Research Paper

\title{
Predictors of Residual T1 High Grade on Re- Transurethral Resection in a Large Multi-Institutional Cohort of Patients with Primary T1 High-Grade/Grade 3 Bladder Cancer
}

\author{
Matteo Ferro ${ }^{\bowtie}$, Giuseppe Di Lorenzo², Carlo Buonerba 2,3 , Giuseppe Lucarelli ${ }^{4}$, Giorgio Ivan Russo ${ }^{5}$, \\ Francesco Cantiello6, Abdal Rahman Abu Farhan 6 , Savino Di Stasi ${ }^{7}$, Gennaro Musi ${ }^{1}$, Rodolfo Hurle ${ }^{8}$, \\ Serretta Vincenzo 9 , Gian Maria Busetto ${ }^{10}$, Ettore De Berardinis10, Sisto Perdonà11, Marco Borghesi12, \\ Riccardo Schiavina ${ }^{12}$, Gilberto L. Almeida13, Pierluigi Bove14, Estevao Lima15,Giovanni Grimaldi 15, Deliu \\ Victor Matei ${ }^{1}$, Francesco Alessandro Mistretta ${ }^{1}$, Nicolae Crisan ${ }^{16}$, Daniela Terracciano ${ }^{17}$, Verze Paolo ${ }^{18}$, \\ Michele Battaglia2 ${ }^{2}$ Giorgio Guazzoni ${ }^{8}$, Riccardo Autorino19, Giuseppe Morgia ${ }^{3}$, Rocco Damiano ${ }^{4}$, Matteo \\ Muto $^{20}$, Roberto La Rocca ${ }^{18}$, Vincenzo Mirone ${ }^{18}$, Ottavio de Cobelli 1,21, Mihai Dorin Vartolomei ${ }^{1,22}$ \\ 1. Division of Urology, European Institute of Oncology, Milan, Italy. \\ 2. Department of Clinical Medicine and Surgery, Federico II University of Naples, Naples, Italy. \\ 3. Istituto Zooprofilattico Sperimentale del Mezzogiorno, Portici, Italy. \\ 4. Department of Emergency and Organ Transplantation, Urology, Andrology and Kidney Transplantation Unit, University of Bari, Bari, Italy. \\ 5. Department of Urology, University of Catania, Catania, Italy. \\ 6. Department of Urology, Magna Graecia University of Catanzaro, Catanzaro, Italy. \\ 7. Urology Unit, Policlinico Tor Vergata, University of Rome, Rome, Italy. \\ 8. Department of Urology, Istituto Clinico Humanitas, Clinical and Research Hospital, Milan, Italy. \\ 9. Division of Urology, University of Palermo, Palermo, Italy. \\ 10. Department of Urology, La Sapienza University of Rome, Rome, Italy. \\ 11. Department of Uro-Gynaecological Oncology, Istituto Nazionale Tumori "Fondazione G. Pascale", IRCCS, Naples, Italy. \\ 12. Department of Urology, University of Bologna, Bologna, Italy. \\ 13. Department of Urology, University of Vale do Itajaí, Itajaí, Brazil. \\ 14. Department of Experimental Medicine and Surgery, Urology Unit, Azienda Policlinico Tor Vergata, Rome, Italy. \\ 15. Department of CUF Urology and Life and Health Sciences Research Institute, School of Medicine, University of Minho, Portugal. \\ 16. Department of Urology, University of Medicine and Pharmacy "Iuliu Hațieganu”, Cluj-Napoca, Romania. \\ 17. Department of Translational Medical Sciences, University of Naples "Federico II", Naples, Italy. \\ 18. Department of Neurosciences, Sciences of Reproduction and Odontostomatology, Urology Unit, University of Naples "Federico II", Naples, Italy. \\ 19. Division of Urology, Virginia Commonwealth University, Richmond, VA, USA. \\ 20. Department of Clinical Medicine and Surgery, Federico II University Medical School of Naples, Italy. \\ 21. University of Milan, Milan, Italy. \\ 22. Department of Cell and Molecular Biology, University of Medicine and Pharmacy, Tirgu Mures, Romania. \\ $\bowtie$ Corresponding author: Matteo Ferro, Division of Urology, European Institute of Oncology - Milan, Italy. Email: matteo.ferro@ieo.it
}

( ) Ivyspring International Publisher. This is an open access article distributed under the terms of the Creative Commons Attribution (CC BY-NC) license (https://creativecommons.org/licenses/by-nc/4.0/). See http://ivyspring.com/terms for full terms and conditions.

Received: 2018.03.17; Accepted: 2018.09.09; Published: 2018.10.20

\begin{abstract}
The aim of this multi-institutional study was to identify predictors of residual high-grade (HG) disease at re-transurethral resection (reTUR) in a large cohort of primary T1 HG/Grade 3 (G3) bladder cancer patients.

A total of 1155 patients with primary T1 HG/G3 bladder cancer from 13 academic institutions that underwent a reTUR within 6 weeks after first TUR were evaluated. Logistic regression analysis was performed to assess the association of predictive factors with residual HG at reTUR.

Residual HG cancer was found in 288 (24.9\%) of patients at reTUR. Patients presenting residual HG cancer were more likely to have carcinoma in situ $(C I S)$ at first resection $(p<0.001)$, multiple tumors $(p=0.02)$, and tumor size larger than $3 \mathrm{~cm}(p=0.02)$. Residual HG disease at reTUR was associated with increased preoperative neutrophil-to-lymphocytes ratio $(N L R)(p=0.006)$ and body mass index
\end{abstract}


$(B M I)>=25 \mathrm{~kg} / \mathrm{m}^{2}$. On multivariable analysis, independent predictors for HG residual disease at reTUR were tumor size $>3 \mathrm{~cm}(\mathrm{OR}=1.37$; $95 \% \mathrm{Cl}$ : 1.02-1.84, $\mathrm{p}=0.03)$, concomitant $\mathrm{CIS}$ (OR 1.92; 95\% Cl: 1.32-2.78, $p=0.001)$, being overweight $(\mathrm{OR}=2.08 ; 95 \% \mathrm{Cl}: 1.44-3.01, \mathrm{p}<0.001)$ and obesity (OR 2.48; 95\% Cl: 1.64-3.77, $\mathrm{p}<0.001$ ).

A reTUR in high grade T1 bladder cancer is mandatory as about $25 \%$ of patients, presents residual high grade disease. Independent predictors to identify patients at risk of residual high grade disease after a complete TUR include tumor size, presence of carcinoma in situ, and BMI $>=25 \mathrm{~kg} / \mathrm{m}^{2}$.

Key words: bladder cancer, neutrophil-to-lymphocytes ratio, re-transurethral resection, high-grade

\section{Introduction}

Transurethral resection of bladder tumor (TUR) is considered the gold standard for the management of non-muscle invasive bladder cancer (NMIBC), followed by adjuvant intravesical therapy according to risk stratification [1,2]. A repeat TUR (reTUR) is now considered an essential step to obtain complete tumor resection and appropriate staging in $\mathrm{T} 1$ stage disease [3].

Most national and international guidelines recommend reTUR[1], mainly due to high prevalence of residual tumor found after reTUR and its clinical implications[4]. Nevertheless, controversy on the topic still exists as some argued that reTUR may not be needed when an adequate first TUR has been performed [5].

The aim of this multi-institutional study was to identify predictors of residual high-grade (HG) disease at reTUR in a large cohort of primary $\mathrm{T} 1$ HG/Grade 3(G3) NMIBC patients.

\section{Material and Methods}

\section{Patient selection and data collection}

Institutional-review-board approval was obtained in each institution. Inclusion criteria were established before data collection: (1) pathological T1 HG/G3 confirmed after first TUR; (2) a reTUR performed within 4 to 6 weeks after a complete first TUR (defined by confirmed presence of muscularis propria on pathology); (3) pretreatment NLR available prior to TUR; (4) history of smoking status and BMI.

Patients with systemic diseases that could interfere with NLR at the time of TUR (such as leukemia, lymphoma, chronic inflammatory diseases, or autoimmune diseases) were excluded. BMI was defined as the weight in kilograms divided by the square of the height in meters $\left(\mathrm{kg} / \mathrm{m}^{2}\right)$, and was categorized in underweight $\left(<18.5 \mathrm{~kg} / \mathrm{m}^{2}\right)$, normal weight (18.5-24.99 $\left.\mathrm{kg} / \mathrm{m}^{2}\right)$, overweight (25-29.99 $\left.\mathrm{kg} / \mathrm{m}^{2}\right)$ and obese $\left(\geq 30 \mathrm{~kg} / \mathrm{m}^{2}\right)$ according to the International Classification of adult underweight, overweight and obesity according to BMI [6].

A total of 1155 HGT1 NMIBC consecutive patients from 13 academic institutions that underwent a reTUR within 6 weeks after first TUR between $1^{\text {st }}$ January 2002 and 31 ${ }^{\text {st }}$ December 2012 were included. Patients who had MIBC at subsequent reTUR were excluded. There was no interim intravesical therapy after initial TUR. Demographical, clinical and pathological data of first and second resection were collected and entered in a computerized database. Histology was performed by experienced uro-pathology at each institution. Tumors were classified histologically using the 1973 World Health Organization (WHO) and tumor, node and metastasis classifications [7]. Protocol of reTUR included tumor scar and base resection, together with the bladder neck (for CIS) and red bladder patches. ReTUR was generally performed by the same urologist who performed the first TUR of bladder tumor [8].

\section{Statistical analysis}

Associations of T1 HG/G3 at reTUR with categorical variables were assessed using $\chi^{2}$ tests while differences in continuous variables were analyzed using $\mathrm{t}$ test after assessing normality of the distribution (Kolmogorov-Smirnov). Logistic regression analysis was performed to assess the association of several predictive factors (age, gender, smoking status, size, multifocality, concomitant CIS, NLR, and BMI) with residual HG at reTUR. All $p$ values were two-sided, and statistical significance was defined as a $\mathrm{p}<0.05$. Statistical analyses were performed using Stata 11.0 statistical software (Stata Corp., College Station, TX, USA).

\section{Results}

\section{Association of HG/G3 at reTUR with clinic and pathological characteristics}

Residual HG disease was found in 288 (24.9\%) of patients after reTUR. Patients with residual HG disease at reTUR were more likely to have concomitant Carcinoma in Situ (CIS) at first resection ( $20.1 \%$ vs. $11.3 \%, \mathrm{p}<0.001)$, multiple tumors $(50.4 \%$ vs. $42.8 \%, \mathrm{p}=0.02)$, and tumor size larger than $3 \mathrm{~cm}(70.5 \%$ vs. $62.7 \%, \mathrm{p}=0.01$ ) and increased pre-treatment (prior 
to initial TUR) neutrophil-to-lymphocytes ratio (NLR) $(57.3 \%$ vs. $47.8 \%, p=0.006)$. In terms of body mass index (BMI) stratification, overweight and obese patients were more likely to have residual HG disease at reTUR $(\mathrm{p}<0.001$, Table 1$)$.

\section{Predictive factors for residual HG disease at reTUR}

On univariable analysis, predictive factors for residual $\mathrm{HG}$ disease at reTUR were size $>3 \mathrm{~cm}(\mathrm{OR}$
1.41, $\mathrm{p}=0.01)$, multifocality (OR 1.35, $\mathrm{p}=0.02)$, concomitant CIS (OR 1.97, p<0.001), NLR >3 (OR 1.46, $\mathrm{p}=0.006$ ) and BMI (overweight and and obese, $\mathrm{p}<0.001$; OR 2.16 and 2.57, respectively). On multivariable analysis, size $>3 \mathrm{~cm}$ (OR 1.37, $\mathrm{p}=0.03$ ), concomitant CIS (OR 1.92, $\mathrm{p}=0.001$ ), overweight (OR $2.08, \mathrm{p}<0.001)$ and obesity (OR 2.48, $\mathrm{p}<0.001)$ status according to BMI remained as significant independent predictors for HG residual disease at reTUR (Table 2).

Table 1. Association of HG/G3 on reTUR with clinical and pathologic characteristics of 1155 patients after primary T1 HG/G3 NMIBC

\begin{tabular}{|c|c|c|c|c|}
\hline & All patients & No tumor/G2 & HG/G3 & p-value \\
\hline Total, n (\%) & 1155 & $867(75.1)$ & $288(24.9)$ & \\
\hline Age Mean (range) & $70.33(46-88)$ & 70.32 & 70.3 & 0.97 \\
\hline \multicolumn{5}{|l|}{ Gender, n (\%) } \\
\hline Male & 957 (82.9) & $715(82.5)$ & $242(84)$ & 0.54 \\
\hline Female & $198(17.1)$ & $152(17.5)$ & $46(16)$ & \\
\hline \multicolumn{5}{|l|}{ Smoking status } \\
\hline never & $328(28.4)$ & $252(29.1)$ & $76(26.4)$ & 0.45 \\
\hline current & $549(47.5)$ & $403(46.4)$ & $146(50.7)$ & \\
\hline former & $278(24.1)$ & $212(24.5)$ & $66(22.9)$ & \\
\hline \multicolumn{5}{|l|}{ Multifocality, n (\%) } \\
\hline single & 639 (55.3) & $496(57.2)$ & $143(49.6)$ & 0.02 \\
\hline multiple & $516(44.7)$ & $371(42.8)$ & $145(50.4)$ & \\
\hline \multicolumn{5}{|l|}{ Size, n (\%) } \\
\hline$<=3 \mathrm{~cm}$ & 408 (35.3) & $323(37.3)$ & 85 (29.5) & 0.01 \\
\hline$>3 \mathrm{~cm}$ & 747 (64.7) & $544(62.7)$ & $203(70.5)$ & \\
\hline \multicolumn{5}{|c|}{ Concomitant carcinoma in situ, $\mathrm{n}(\%)$} \\
\hline No & $999(86.5)$ & 769 (88.7) & $230(79.9)$ & $<0.001$ \\
\hline Yes & $156(13.5)$ & $98(11.3)$ & $58(20.1)$ & \\
\hline \multicolumn{5}{|l|}{ NLR, n (\%) } \\
\hline$<=3$ & $575(49.8)$ & $452(52.1)$ & $123(42.7)$ & 0.006 \\
\hline$>3$ & $580(50.2)$ & $415(47.8)$ & $165(57.3)$ & \\
\hline BMI normal & $337(29.2)$ & $285(32.9)$ & $52(18.1)$ & $<0.001$ \\
\hline underweight & $24(2.1)$ & $22(2.5)$ & $2(0.7)$ & \\
\hline overweight & $534(46.2)$ & $383(44.2)$ & $151(52.4)$ & \\
\hline obese & $260(22.5)$ & $177(20.4)$ & $83(28.8)$ & \\
\hline
\end{tabular}

TUR: transurethral resection of bladder tumor, NMIBC: non-muscle invasive bladder cancer; NLR: neutrophil-to-lymphocytes ratio, BMI: body mass index

Table 2. Univariate and multivariate logistic regression analyses for predicting residual high grade disease at reTUR in 1155 patients with primary TIHG/G3 NMIBC.

\begin{tabular}{|c|c|c|c|c|c|c|}
\hline \multirow{3}{*}{$\begin{array}{l}\text { Preoperative prognostic } \\
\text { factors }\end{array}$} & \multicolumn{6}{|c|}{ HG/G3 on reTUR } \\
\hline & \multicolumn{3}{|c|}{ Univariate } & \multicolumn{3}{|c|}{ Multivariate } \\
\hline & OR & $95 \% \mathrm{CI}$ & $\mathrm{p}$ & OR & $95 \% \mathrm{CI}$ & $\mathrm{p}$ \\
\hline Age (continuous) & 0.99 & $0.98-1.01$ & 0.97 & 1 & $0.98-1.01$ & 0.86 \\
\hline Gender (male vs. female) & 0.89 & $0.62-1.28$ & 0.54 & 0.86 & $0.59-1.25$ & 0.44 \\
\hline Smoking status & \multicolumn{6}{|c|}{ Never smoker is reference } \\
\hline Current smoker & 1.2 & $0.87-1.65$ & 0.26 & 1.14 & $0.82-1.59$ & 0.42 \\
\hline Former smoker & 1.03 & $0.7-1.5$ & 0.86 & 1.13 & $0.76-1.69$ & 0.53 \\
\hline Multifocality (Yes. Vs. no) & 1.35 & $1.03-1.77$ & 0.02 & 1.26 & $0.96-1.66$ & 0.09 \\
\hline Size $>3 \mathrm{~cm}$ vs. $<=3 \mathrm{~cm}$ & 1.41 & $1.06-1.89$ & 0.01 & 1.37 & $1.02-1.84$ & 0.03 \\
\hline Concomitant CIS (Yes vs. no) & 1.97 & $1.38-2.82$ & $<0.001$ & 1.92 & $1.32-2.78$ & 0.001 \\
\hline NLR $>3$ vs. $<=3$ & 1.46 & $1.11-1.91$ & 0.006 & 1.12 & $0.83-1.5$ & 0.44 \\
\hline BMI & \multicolumn{6}{|c|}{ Normal weight is reference } \\
\hline underweight & 0.49 & $0.11-2.18$ & 0.35 & 0.53 & $0.12-2.36$ & 0.4 \\
\hline overweight & 2.16 & $1.52-3.06$ & $<0.001$ & 2.08 & $1.44-3.01$ & $<0.001$ \\
\hline obese & 2.57 & $1.73-3.81$ & $<0.001$ & 2.48 & $1.64-3.77$ & $<0.001$ \\
\hline
\end{tabular}

TURBT: transurethral resection of bladder tumor, OR: Odds ratio, CI: Confidence interval 


\section{Discussion}

We showed that residual HG disease at re-TUR was reported in one out of four patients with initial T1HG NMIBC. It was associated with worse clinical characteristics such as increased BMI and increased pretreatment NLR and worse pathological features such as multifocality, tumor size $>3 \mathrm{~cm}$ and presence of concomitant CIS at first TUR in a cohort of patients with primary T1 HG/G3 NMIBC. Moreover, independent predictors for residual HG disease at reTUR were size $>3 \mathrm{~cm}$, presence of concomitant CIS and BMI $>=25 \mathrm{~kg} / \mathrm{m}^{2}$.

In a mono-center study, multiplicity, T1 and HG in the initial TUR were shown to be independent risk factors for residual tumors at reTUR [9]. In another study that included 179 patients with NMIBC, a high risk of recurrence according to the EAU risk score classification at the initial TUR and multifocality were associated with higher rates of residual tumor [10].

In our retrospective study, we showed that in patients wth high-risk tumors (i.e. HGT1), BMI may contribute to identify patients that could have residual HG disease at reTUR.

Certainly, a complete and correctly performed TUR is essential to achieve good prognosis as the residual tumor rate at reTUR can be as high as $47 \%$ (95\% CI: 0.41-0.53) [11]. Re-TURBT is indicated and should be routinely performed in T1 NMIBC also to reduce the risk of under-staging and missing MIBC[3]. The presence of a high-risk cancer at first TUR was shown to be an independent risk factor for residual disease at reTUR in several studies [10,12]. Similarly, concomitant CIS also significantly correlated with incidence of residual tumor in a prospective study that included 52 patients, while the absence of muscularis propria in the primary TUR specimen was associated with upstaging to MIBC [13]. In our cohort, one of the inclusion criteria was the presence of muscle tissue at the first TUR. Takaoka et al. showed that CIS was also a risk factor for residual tumors at reTUR in a cohort that included HGT1 patients [14].Tumor multiplicity at the first resection was found to be an independent risk factor for stage pT1 or worse tumor at re-TUR in a multi-institutional study that included Japanese patients [15]. Moreover, one study that included 188 African patients with T1 NMIBC found that male gender along with multifocality are risk factors for residual tumors at reTUR [16].

Liu et al. found out that patients with altered p53 and E-cadherin expression were more likely to have residual tumors [17]. Lodde et al found that a positive cytology prior to second TUR was associated with positive re- TUR[18].

One recently published meta-analysis showed a nonlinear positive relationship between BMI and BC risk, with a a $5 \mathrm{~kg} / \mathrm{m}^{2}$ increment of BMI being associated with a $3.1 \%$ increase of bladder cancer risk [19]. Patients diagnosed with clinical HGT1 urothelial carcinoma of the bladder who are obese have worse cancer specific outcomes compared to their non-obese counterparts [20]. To our knowledge, the study presented here is the first that shows that increased BMI is associated with residual HG disease after a complete TUR. Although the potential etiopathogenetic association with obesity is intriguing, we acknowledge that this finding may be due to the difficulty of transurethral resection in case of overweight and obese patients, as was shown also in case of transurethral resection of the prostate [21].

A higher NLR was reported to be associated with T1 vs. Ta tumors tumor stage at the time of TUR (mean 3.9 vs. 2.5)[22].In our cohort, NLR was a predictor of residual HG disease at reTUR at univariable analysis but did not retain its significance at multivariable analysis. Indeed, in T1 vs. Ta NMIBC inflammatory markers levels are higher, and associated with progression [23] and recurrence [24]. These findings have also been replicated in patients with MIBC $[25,26]$. However, prospectively collected data showed that pretreatment NLR was not associated with overall survival in MIBC patients after radical cystectomy, which is consistent with our findings [27].

The limitations of the present study are those typical of retrospective studies, including the presence of a selection bias, as well as heterogenous surgeon expertise and surgical technique. Furthermore, patients were not assessed for consumption of steroids, presence of infection or thromboembolism, which may affect NLR, nor were tumor location and lymph-vascular invasion included in the multivariable analysis. Despite these limitations, we believe that our study provides evidence indicating a potential association between obesity and risk of residual disease after TUR, which should be further explored in order to assess its potential practical clinical implications as well as its etiopathogenetic basis.

\section{Conclusion}

Re-TURBT should be routinely performed in T1 NMIBC to reduce the risk of under-staging and missing MIBC. A re-TUR in high grade T1 bladder cancer is mandatory considering that about $25 \%$ of patients present residual high grade disease. Independent predictors to identify patients at risk of residual high grade disease after a complete TUR are tumor size, presence of carcinoma in situ, and BMI $>=25 \mathrm{~kg} / \mathrm{m}^{2}$. 


\section{Acknowledgements}

This study was supported by "Fondazione Muto", Naples-Italy. M.D.V is supported by the Scholarship Foundation of the Republic of Austria OeAD and by the EUSP Scholarship - European Association of Urology.

\section{Conflict of interest}

The authors declare that they have no conflict of interest.

\section{References}

1. Richterstetter M, Wullich B, Amann K, Haeberle L, Engehausen DG, Goebell PJ, Krause FS. The value of extended transurethral resection of bladder tumour (TURBT) in the treatment of bladder cancer. BJU Int. 2012; 110: E76-79. doi: 10.1111/j.1464-410X.2011.10904.x.

2. Babjuk M, Bohle A, Burger M, Comperat E, Kaasinen E, Palou J, Rouprêt M, van Rhijn BW, Shariat SF, Sylvester R, Zigeuner R. Guidelines on non-muscle-invasive Bladder Cancer (Ta,T1 and CIS). EAU Guidelines. European Association of Urology; 2015.

3. Naselli A, Hurle R, Paparella S, Buffi NM, Lughezzani G, Lista G, Casale P, Saita A, Lazzeri M, Guazzoni G. Role of Restaging Transurethral Resection for T1 Non-muscle invasive Bladder Cancer: A Systematic Review and Meta-analysis. Eur Urol Focus. 2017; . doi: 10.1016/j.euf.2016.12.011.

4. Schwaibold HE, Sivalingam S, May F, Hartung R. The value of a second transurethral resection for T1 bladder cancer. Bju Int. 2006; 97: 1199-201. doi: 10.1111/j.1464-410X.2006.06144.x.

5. Brausi MA. Challenging the EAU Guidelines Regarding Early Repeat Transurethral Resection. Eur Urol Suppl. 2011; 10: E5-7. doi: 10.1016/j.eursup.2011.04.003.

6. Flegal KM, Carroll MD, Ogden CL, Curtin LR. Prevalence and trends in obesity among US adults, 1999-2008. JAMA. 2010; 303: 235-41. doi: 10.1001/jama.2009.2014

7. Balan D, Martha O, Chibelean CB, Tataru S, Voidezan S, Sin A, Matei VD, Vartolomei MD, Lucarelli G, Cioffi A, Del Giudice F, De Berardinis E, Borda A, et al. Comparison of 10-year overall survival between patients with G1 and G2 grade Ta bladder tumors. Medicine (Baltimore). 2018; 97: e0522. doi: 10.1097/MD.0000000000010522.

8. Del Zingaro M, Bruno R, Nunzi E, Porena M, Mearini L. First and second transurethral resections in intermediate-high risk bladder cancer: impact of the surgeon's volume on the recurrence and progression of primary bladder cancer. Minerva Urol E Nefrol Ital J Urol Nephrol. 2016; 68: 194-203.

9. Cao M, Yang G, Pan J, Sun J, Chen Q, Chen Y, Chen H, Xue W. Repeated transurethral resection for non-muscle invasive bladder cancer. Int J Clin Exp Med. 2015; 8: 1416-9.

10. Hartinger J, Häußermann R, Olbert P, Hofmann R, Hegele A. [Predictors for presence of residual tumor in follow-up transurethral resection of bladder tumors: single center results]. Urol Ausg A. 2013; 52: 557-61. doi: 10.1007/s00120-012-3109-x.

11. Vianello A, Costantini E, Del Zingaro M, Bini V, Herr HW, Porena M. Repeated White Light Transurethral Resection of the Bladder in Nonmuscle-Invasive Urothelial Bladder Cancers: Systematic Review and Meta-Analysis. J Endourol. 2011; 25: 1703-12. doi: 10.1089/end.2011.0081.

12. Husillos Alonso A, Rodríguez Fernández E, Herranz Amo F, López López E, Aragón Chamizo J, Ramírez Martínez D, Durán Merino R, Hernández Fernández $\mathrm{C}$. The need for re-TUR of the bladder in non-muscle invasive bladder cancer: risk factors of tumor persistence in re-TUR specimens. Minerva Urol E Nefrol Ital J Urol Nephrol. 2014; 66: 233-40

13. Gill TS, Das RK, Basu S, Dey RK, Mitra S. Predictive factors for residual tumor and tumor upstaging on relook transurethral resection of bladder tumor in non-muscle invasive bladder cancer. Urol Ann. 2014; 6: 305-8. doi: 10.4103/0974-7796.140990.

14. Takaoka E, Matsui $Y$, Inoue T, Miyazaki J, Nakashima M, Kimura T, Oikawa T, Kawai K, Yoshimura K, Habuchi T, Ogawa O, Nishiyama H. Risk factors for intravesical recurrence in patients with high-grade $\mathrm{T} 1$ bladder cancer in the second TUR era. Jpn J Clin Oncol. 2013; 43: 404-9. doi: 10.1093/jico/hyt016.

15. Kamiya N, Suzuki H, Suyama T, Kobayashi M, Fukasawa S, Sekita N, Mikami K, Nihei N, Naya Y, Ichikawa T. Clinical outcomes of second transurethral resection in non-muscle invasive high-grade bladder cancer: a retrospective, multi-institutional, collaborative study. Int J Clin Oncol. 2017; 22: 353-8. doi: 10.1007/s10147-016-1048-z.

16. Turk H, Un S, Isoglu CS, Karabicak M, Ergani B, Yoldas M, Tarhan H, Zorlu F. Factors that predict residual tumors in re-TUR patients. Afr J Urol. 2016; 22: 67-70. doi: 10.1016/j.afju.2015.07.004

17. Liu W, Qi L, Zu X, Li Y, He W, Tong S, Chen M. A preoperative marker panel for the prediction of residual tumor and the decision making for repeat transurethral resection. Urol Oncol. 2015; 33: 165.e9-14 doi: 10.1016/j.urolonc.2015.01.006.

18. Lodde M, Mayr R, Martini T, Comploj E, Palermo S, Trenti E, Hanspeter E, Fritsche HM, Mian C, Pycha A. Positive urine cytology and carcinoma in situ prior to second transurethral resection of the bladder correlate with positive second resection histology and the need for subsequent cystectomy. World J Urol. 2012; 30: 841-6. doi: 10.1007/s00345-012-0975-1.

19. Zhao L, Tian X, Duan X, Ye Y, Sun M, Huang J. Association of body mass index with bladder cancer risk: a dose-response meta-analysis of prospective cohort studies. Oncotarget. 2017; 8: 33990-4000. doi: 10.18632/oncotarget.16722.

20. Kluth LA, Xylinas E, Crivelli JJ, Passoni N, Comploj E, Pycha A, Chrystal J, Sun M, Karakiewicz PI, Gontero P, Lotan Y, Chun FK-H, Fisch M, et al. Obesity is associated with worse outcomes in patients with T1 high grade urothelial carcinoma of the bladder. J Urol. 2013; 190: 480-6. doi: 10.1016/j.juro.2013.01.089.

21. Gacci M, Sebastianelli A, Salvi M, De Nunzio C, Tubaro A, Vignozzi L, Corona G, McVary KT, Kaplan SA, Maggi M, Carini M, Serni S. Central obesity is predictive of persistent storage lower urinary tract symptoms (LUTS) after surgery for benign prostatic enlargement: results of a multicentre prospective study. BJU Int. 2015; 116: 271-7. doi: 10.1111/bju.13038.

22. Tazeh NN, Canter DJ, Damodaran S, Rushmer T, Richards KA, Abel EJ, Jarrard DF, Downs TM. Neutrophil to Lymphocyte Ratio (NLR) at the Time of Transurethral Resection of Bladder Tumor: A Large Retrospective Study and Analysis of Racial Differences. Bladder Cancer Amst Neth. 2017; 3: 89-94. doi: 10.3233/BLC-160085

23. Martha O, Porav-Hodade D, Bălan D, Tătaru OS, Sin A, Chibelean CB, Vartolomei MD. Easily Available Blood Test Neutrophil-To-Lymphocyte Ratio Predicts Progression in High-Risk Non-Muscle Invasive Bladder Cancer. Rev Romana Med Lab. 2017; 25: 181-9. doi: 10.1515/rrlm-2017-0016.

24. Mbeutcha A, Shariat SF, Rieken M, Rink M, Xylinas E Seitz C, Lucca I, Mathieu R, Rouprêt M, Briganti A, Karakiewicz PI, Klatte T. Prognostic significance of markers of systemic inflammatory response in patients with non-muscle-invasive bladder cancer. Urol Oncol. 2016; 34: 483.e17-483.e24. doi: 10.1016/j.urolonc.2016.05.013.

25. Masson-Lecomte A, Rava M, Real FX, Hartmann A, Allory Y, Malats N. Inflammatory biomarkers and bladder cancer prognosis: a systematic review. Eur Urol. 2014; 66: 1078-91. doi: 10.1016/j.eururo.2014.07.033.

26. Lucca I, Jichlinski P, Shariat SF, Rouprêt M, Rieken M, Kluth LA, Rink M, Mathieu R, Mbeutcha A, Maj-Hes A, Fajkovic H, Briganti A, Seitz C, et al. The Neutrophil-to-lymphocyte Ratio as a Prognostic Factor for Patients with Urothelial Carcinoma of the Bladder Following Radical Cystectomy: Validation and Meta-analysis. Eur Urol Focus. 2016; 2: 79-85. doi: 10.1016/j.euf.2015.03.001.

27. Ojerholm E, Smith A, Hwang W-T, Baumann BC, Tucker KN, Lerner SP, Mamtani R, Boursi B, Christodouleas JP. Neutrophil-to-Lymphocyte Ratio as a Bladder Cancer Biomarker: Assessing Prognostic and Predictive Value in SWOG 87 10. Cancer. 2017; 123: 794-801. doi: 10.1002/cncr.30422. 